\title{
CALIDAD DE VIDA Y CONDUCTAS ALIMENTARIAS DE RIESGO EN LA PREADOLESCENCIA
}

\section{QUALITY OF LIFE AND EATING BEHAVIORS IN PREADOLESCENCE}

\author{
Alfonso Urzúa M., Francisca Avendaño H., Stefanía Díaz C., Daniela Checura \\ Área Psicología de la Salud \\ Escuela de Psicología, Universidad Católica del Norte. Antofagasta, Chile.
}

\begin{abstract}
The relationship between the quality of life and eating behaviors in preadolescents between 10 to 13 years of age was determined by a cross-sectional design, Was assessed data from 702 participants (mean age of 11.5 years, 375 women and 327 men) recruited through KIDSCREEN-52 to measure quality of life and EAT-26 to assess eating behaviors. We found that weight control and body image that leads to twins is related to lower quality of life, particularly in the dimension of physical bullying, school environment, emotional state and mood, self-perceived autonomy and economic resources. The findings, as well as evidence that these behaviors are increasingly prevalent at younger ages, warrant the development of early preventive interventions in the field of eating behaviors. Key words: quality of life, eating behaviors, preadolescence.
\end{abstract}

Este trabajo fue recibido el 13 de Abril de 2010 y aceptado para ser publicado el 20 de Agosto de 2010.

\section{INTRODUCCIÓN}

La Organización Mundial de la Salud (OMS) considera a la adolescencia como el período de tiempo comprendido entre los 10 y 19 años. Como una sub etapa se destaca la preadolescencia, periodo comprendido entre los 9 y 13 años, que marca el inicio de la pubertad (1).

En esta etapa se producen cambios endocrinos, morfológicos y psicológicos propios de la adolescencia, convirtiéndose en una etapa vulnerable debido a las transformaciones biológicas puberales, demandas psicosociales, inestabilidad emocional y conflictos en la identidad personal relacionados con la búsqueda de un cuerpo cada vez más delgado (2) y al deber integrar adecuadamente en la construcción de su ser una nueva imagen corporal, la que muchas veces se ve encontrada con los modelos impuestos por la sociedad.

Los preadolescentes y adolescentes manifiestan preferencias hacia determinadas estéticas impuesta por la sociedad contemporánea, haciendo que los más jóvenes presenten una preocupación excesiva por su cuerpo y lleven a cabo conductas alimentarias de riesgo (CAR) (3), entendidas como aquellas manifestaciones que no cumplen con los criterios de un Trastorno de la Conducta Alimentaria, pero que son conductas características de los mismos, tales como práctica de dietas, atracones, conductas purgativas y miedo a engordar (4).

En Chile, estudios realizados en población adolescente sobre prevalencias de CAR para los trastornos de la conducta alimentaria (TCA) han reportado diversos porcentajes que oscilan desde un 7.4 a un $18 \%(2,5-7)$.

El estudio de las CAR se ha vuelto relevante, no sólo por que estas favorecen la aparición de enfermedades crónicas degenerativas, si no también por el cómo estas afectarían el bienestar y la percepción de la calidad de vida $(\mathrm{CV})$ de la población preadolescente.

En este contexto, el estudio de la CV cobra importancia debido a su aspecto multidimensional, abarcando aspectos del funcionamiento físico, emocional, social, percepción de bienestar y salud en general. En términos operativos, puede definirse como el nivel de bienestar derivado de la evaluación que la persona realiza de diversos dominios de su vida, considerando el impacto que en éstos tiene su estado de salud (8). Esta evaluación que hace cada persona tiene un carácter eminentemente 
subjetivo y está influenciada directamente por las características propias de la persona que evalúa, siendo la etapa del desarrollo evolutivo una de estas.

Pese a la importancia que tendría la investigación en esta área, en población escolar y preadolescente, no se ha encontrado estudios en que se relacione las CAR con la CV. Por otro lado, datos relacionados con intervención dirigidas a ésta población también son insuficientes, puesto que son grupos tradicionalmente más alejados de los centros primarios de atención de salud y que no han sido priorizados como grupo objetivo de investigaciones.

Por ello se hace relevante abordar esta etapa a fin de prevenir el surgimiento de diferentes problemáticas concernientes tanto a la salud mental como física que eventualmente pudiesen generar consecuencias relacionadas en la percepción de la $\mathrm{CV}$ de los preadolescentes.

La presente investigación tuvo como objetivo analizar la relación entre las variables calidad de vida y conductas alimentarias de riesgo en preadolescentes chilenos de entre 10 y 13 años de edad. Adicionalmente de manera específica se analizó las diferencias en cada una de estas variables y en la relación entre ambas dadas por el sexo, el rango de edad y el tipo de establecimiento educacional.

\section{SUJETOS Y MÉTODO Participantes}

Se obtuvo una muestra intencionada estratificada, intentando cuidar una similar distribución de sexo, tipos de establecimiento y edad. Participaron 702 pre adolescentes procedentes de seis establecimientos educacionales de la ciudad de Antofagasta: dos municipales (37.9\%), dos subvencionados (38.6\%) y dos particulares $(23,5 \%)$. Las edades de los sujetos encuestados fueron de $10(20.4 \%), 11(26.5 \%), 12(28.5 \%)$ y 13 años $(24.6 \%)$ de edad. Fueron encuestados 375 mujeres (53.4\%) y 327 hombres $(46.6 \%)$.

\section{Procedimientos}

Una vez obtenida la aprobación del Comité de Bioética de la Universidad Católica del Norte y de las autorizaciones necesarias de las direcciones de los establecimientos, se recolectaron los datos considerándose como único criterio de inclusión el tener una edad entre 10 a 13 años inclusives.

Las evaluaciones se aplicaron en horario de clases a cursos completos, después del respectivo asentimiento informado de los participantes y previo el consentimiento de sus padres. Los cuestionarios fueron respondidos en un lapso de entre 20 a 45 minutos.
Una vez completados los cuestionarios, los datos fueron ingresados a una planilla construida en SPSS 12.0.

\section{Instrumentos}

Para medir la calidad de vida se utilizó el cuestionario KIDSCREEN-52 y para evaluar las conductas alimentarias de riesgo, la escala EAT-26.

El cuestionario KIDSCREEN-52 es un instrumento de autoreporte de la salud y el bienestar de niños y adolescentes entre los 8 y los 18 años. Mide 10 dimensiones de CVRS permitiendo obtener un puntaje entre 1 a 100 , en donde un mayor puntaje implica una mayor calidad de vida: bienestar físico ( 5 ítems) el cual, recoge los niveles de actividad física, energía y buena condición física del chico/a; bienestar psicológico (6 ítems) recoge el bienestar psicológico del chico/a, incluidas las emociones positivas y la satisfacción con la vida; estado de ánimo (7 ítems) el cual, recoge las experiencias negativas, los estados de ánimo depresivos y sensaciones de estrés; autopercepción (5 ítems) incluye la percepción del chico/a sobre sí mismo/a, la apariencia física y la satisfacción relacionada con ellos; autonomía (5 ítems) el cual, recoge las oportunidades de disponer de su tiempo de ocio; Relación con los padres y vida familiar (6 ítems) examina la relación con los padres y la atmósfera familiar del chico/a; amigos y apoyo social (6 ítems) recoge la naturaleza de las relaciones sociales del chico/a con otros; entorno escolar ( 6 ítems) el cual, analiza la percepción del chico/a sobre su aptitud para el aprendizaje, concentración y sensaciones sobre la escuela; aceptación social (Bullying) (3 ítems) recoge los sentimientos de rechazo por parte de los compañeros; y recursos económicos (3 ítems) el cual, evalúa la percepción sobre la capacidad financiera familiar.

Se ha reportado buenas propiedades psicométricas en tanto validez y confiabilidad, en su versión en inglés para 13 países europeos (9) y en su versión en castellano (10). Para el presente estudio se encontró una confiabilidad, expresada a través del estadístico alfa de Cronbach, de .81 para la escala total. El test de Actitudes Alimentarias (EAT-26) tiene por objetivo identificar, a través del autoreporte, síntomas y preocupaciones características de los trastornos alimentarios en muestras no clínicas. Sus puntajes oscilan de 0 a 78 puntos, en donde mayor puntaje implica mayor riesgo. Es considerado una de las pruebas estandarizadas más utilizadas en el ámbito de los trastornos alimentarios, ya que posee altos índices de confiabilidad y validez (Corada L, Montedónico A, Wenk Estudio del aporte de un instrumento - test de actitudes alimentarias EAT-26), en la evaluación de cambios en adolescentes sometidos a un programa de prevención 
de obesidad. (Tesis para optar al título de psicólogo, Universidad de Chile. 2007).

Los ítems de la prueba se agrupan en 3 factores: El factor 1 Dieta (13 preguntas, con un puntaje máximo de 39 puntos), se relaciona con la evitación de alimentos que engordan y la preocupación por estar delgado(a), el factor 2 Bulimia (06 preguntas, con un puntaje máximo de 18 puntos) y preocupación por la comida refleja pensamientos sobre la comida e indicadores de bulimia, y el factor 3 control oral (07 preguntas, con un puntaje máximo de 21 puntos) esta relacionado con el autocontrol en el comer y en el percibir presión desde los otros por aumentar de peso. El obtener un puntaje total igual o superior a 20 se asocia a la presencia de actitudes y conductas de riesgo de un desorden alimentario.

La escala en castellano presenta buenas propiedades psicométricas con un Alfa de Cronbach de .86 para la escala total (11). En la presente aplicación se obtuvo un alfa de Cronbach de .76 para la escala total.

\section{Análisis estadístico}

Se calcularon las medidas de tendencia central para las dimensiones evaluadas, estratificando el análisis por sexo, rango de edad y tipo de establecimiento, procediéndose posteriormente a comparar las medias para cada dimensión según sexo, tipo de colegio y rango de edad a través de pruebas t de Student para muestras independientes o ANOVA de un factor, dependiendo del requerimiento de las variables. Las correlaciones se realizaron a través del coeficiente de correlación de Pearson. Se calculó para todas las relaciones la magnitud del tamaño del efecto.

\section{RESULTADOS \\ Participantes}

El promedio de edad para el grupo total correspondió a 11.57 años $(\mathrm{DE}=1.07)$. En las mujeres la media de edad fue de 11.55 años $(\mathrm{DE}=1.08)$ y en los hombres de 11.61 años (DE=1.07). En la tabla 1 se puede observar las frecuencias de sexo y edad segmentadas por tipo de establecimiento.

\section{Actitudes Alimentarias}

En las tablas 2 y 3 se muestran las medias encontradas por dimensión del instrumento EAT-26 segregadas por sexo, rango de edad y tipo de establecimiento.

\section{TABLA 1}

Distribución de los participantes según sexo y edad por tipo de establecimiento.

\begin{tabular}{|lcccc|}
\hline $\begin{array}{l}\text { Tipo de } \\
\text { establecimiento }\end{array}$ & $\begin{array}{c}\text { Mujeres } \\
\mathbf{( 3 7 5 )}\end{array}$ & $\begin{array}{c}\text { Sexo } \\
\mathbf{( 3 2 7 )}\end{array}$ & $\begin{array}{c}\mathbf{1 0 - 1 1} \\
\mathbf{( 3 2 9 )}\end{array}$ & $\begin{array}{c}\mathbf{1 2}-\mathbf{1 3} \\
\mathbf{( 3 7 3 )}\end{array}$ \\
\hline Municipal (266) & $154(57.9 \%)$ & $112(42.1 \%)$ & $123(46.2 \%)$ & $143(53.8 \%)$ \\
Subvencionado (271) & $135(49.8 \%)$ & $136(50.2 \%)$ & $133(49.1 \%)$ & $138(50.9 \%)$ \\
Particular (165) & $86(52.1 \%)$ & $79(47.9 \%)$ & $73(44.2 \%)$ & $92(55.8 \%)$ \\
\hline
\end{tabular}

TABLA 2

Medidas de tendencia central dimensiones del EAT- 26 por sexo y edad.

\begin{tabular}{|c|c|c|c|c|c|}
\hline & \multirow[b]{2}{*}{$\begin{array}{c}\text { Total } \\
(702) \\
X \pm D E\end{array}$} & \multicolumn{2}{|c|}{ Sexo } & \multicolumn{2}{|c|}{ Rango de Edad } \\
\hline & & $\begin{array}{c}\text { Mujeres } \\
(375) \\
X \pm D E\end{array}$ & $\begin{array}{c}\text { Hombres } \\
(327) \\
\mathrm{X} \pm \mathrm{DE}\end{array}$ & $\begin{array}{c}10-11 \\
(329) \\
X \pm D E\end{array}$ & $\begin{array}{c}12-13 \\
(373) \\
X \pm D E\end{array}$ \\
\hline Control Oral & $4.50+3.65$ & $4.77+3.77$ & $4.19+3.50$ & $4.88+3.93$ & $4.16+3.37$ \\
\hline Dieta & $7.2+6.24$ & $7.67+6.60$ & $6.26+5.72$ & $8.30+6.70$ & $5.89+5.58$ \\
\hline Bulimia & $1.72+2.23$ & $1.77+2.24$ & $1.65+2.23$ & $2.08+2.43$ & $1.39+2.00$ \\
\hline Riesgo total & $13.23+9.47$ & $14.21+9.83$ & $12.10+8.92$ & $15.26+10.15$ & $11.44+8.44$ \\
\hline
\end{tabular}


Se encontró que la media de las mujeres fue significativamente mayor que la de los hombres en la dimensiones control oral $(\mathrm{t}=2.12(697.02), \mathrm{p}<.05)$, dieta $(\mathrm{t}=3.03(699.97), \mathrm{p}<.01)$ y riesgo total $(\mathrm{t}=2.99(698.87)$, $\mathrm{p}<.01)$.

Los participantes entre 10 y 11 años tuvieron medias superiores a los que se encuentran en el tramo de edad de 12 a 13, siendo estas diferencias estadísticamente significativas en las dimensiones control oral $(\mathrm{t}=2.60(650.33), \mathrm{p}<.01)$, dieta $(\mathrm{t}=5.14(640.57) ; \mathrm{p}<.01)$, bulimia $(\mathrm{t}=4.05(636.84) ; \mathrm{p}<.01)$ y en la de riesgo total $(\mathrm{t}=5.38(640.14), \mathrm{p}<.01)$.

Se encontraron diferencias en la dimensión control oral en los preadolescentes que estudian en establecimientos subvencionados, los cuales tienen medias significativamente más altas que los que estudian en establecimientos municipales $(\mathrm{F}(2.70)=3.27, \mathrm{p}<.05)$ $\mathrm{y}$ en el riesgo total, en donde existieron diferencias significativas entre los preadolescentes estudiantes de establecimientos particulares y subvencionados, siendo superior en éstos últimos $(\mathrm{F}(2.70)=3.42, \mathrm{p}<.05)$.

\section{Calidad de vida}

Las tablas 4 y 5 contienen las medias encontradas en cada una de las dimensiones de CV segregadas por sexo, rango de edad y tipo de establecimiento.

TABLA 3

Medidas de tendencia central dimensiones del EAT- 26 por tipo de establecimiento.

\begin{tabular}{|c|c|c|c|}
\hline & $\begin{array}{l}\text { Municipal } \\
(266) \\
X \pm D E\end{array}$ & $\begin{array}{c}\text { Tipo de establecimiento } \\
\text { subvencionado } \\
(271) \\
\mathrm{X} \pm \mathrm{DE}\end{array}$ & $\begin{array}{c}\text { Particular } \\
\text { (165) } \\
\text { X } \pm \text { DE }\end{array}$ \\
\hline Control Oral & $4.16+3.56$ & $4.93+3.73$ & $4.34+3.63$ \\
\hline Dieta & $6.66+5.81$ & $7.73+6.68$ & $6.44+6.10$ \\
\hline Bulimia & $1.87+2.41$ & $1.72+2.22$ & $1.44+1.93$ \\
\hline Riesgo total & $12.69+9.01$ & $14.38+9.85$ & $12.21+9.42$ \\
\hline
\end{tabular}

\section{TABLA 4}

Medidas de tendencia central dimensiones del KIDS-52 por sexo y edad.

\begin{tabular}{|c|c|c|c|c|c|}
\hline & \multirow[b]{2}{*}{$\begin{array}{c}\text { Total } \\
(702) \\
X \pm D E\end{array}$} & \multicolumn{2}{|c|}{ Sexo } & \multicolumn{2}{|c|}{ Edad } \\
\hline & & $\begin{array}{c}\text { Mujeres } \\
(375) \\
\mathrm{X}+\mathrm{DF}\end{array}$ & $\begin{array}{c}\text { Hombres } \\
(327) \\
\mathrm{X}+\mathrm{DF}\end{array}$ & $\begin{array}{c}10-11 \\
(329) \\
X+D F\end{array}$ & $\begin{array}{c}12-13 \\
(373) \\
X+D F\end{array}$ \\
\hline Bienestar físico & $53.80+28.64$ & $49.62+21.36$ & $58.60+34.60$ & $56.66+32.52$ & $51.29+24.49$ \\
\hline Bienestar Psicológico & $57.70+22.04$ & $57.88+24.13$ & $57.49+19.40$ & $59.11+22.78$ & $56.45+21.32$ \\
\hline Estado emocional y ánimo & $34.56+25.33$ & $36.61+28.41$ & $32.22+21.04$ & $33.69+24.71$ & $35.33+25.86$ \\
\hline Autopercepción & $44.00+15.49$ & $44.49+16.31$ & $43.44+14.49$ & $44.17+18.74$ & $43.85+11.92$ \\
\hline Autonomía & $51.22+16.76$ & $50.86+17.24$ & $51.62+16.22$ & $51.29+15.77$ & $51.16+17.62$ \\
\hline $\begin{array}{l}\text { Relación con los padres } \\
\text { y vida familiar }\end{array}$ & $57.63+30.81$ & $58.08+32.24$ & $57.13+29.12$ & $56.43+25.34$ & $58.70+34.93$ \\
\hline Recursos económicos & $46.74+11.43$ & $47.31+11.88$ & $46.09+10.86$ & $46.94+11.56$ & $46.56+11.32$ \\
\hline Amigos y apoyo social & $59.13+32.21$ & $57.34+26.11$ & $61.18+37.96$ & $59.86+34.43$ & $58.48+30.16$ \\
\hline Entorno escolar & $57.67+31.11$ & $59.49+33.95$ & $55.59+27.41$ & $60.68+33.93$ & $55.02+28.18$ \\
\hline Bullying & $23.67+10.61$ & $22.83+9.87$ & $24.63+11.35$ & $24.11+11.28$ & $23.28+9.99$ \\
\hline
\end{tabular}


Los hombres presentan medias significativamente mayores que las mujeres en las dimensiones bienestar físico $(\mathrm{t}=4.07$ (527.89), $\mathrm{p}<.01$ y bullying $(\mathrm{t}=-2.24(700)$, $\mathrm{p}<.05)$, en tanto en la dimensión estado emocional y ánimo, la media de las mujeres es significativamente superior a la de los hombres ( $\mathrm{t}=2.30(700) ; \mathrm{p}<.01)$.
Los preadolescentes entre 10 y 11 años, tuvo una media significativamente más alta que los de 12 a 13 años en las dimensiones de bienestar físico ( $\mathrm{t}=2.44$ (604.80), $\mathrm{p}<.05)$ y entorno escolar $(\mathrm{t}=2.39(639.72), \mathrm{p}<.05)$.

La media de los establecimientos particulares fue significativamente mayor que la de los municipales y

\section{TABLA 5}

Medidas de tendencia central dimensiones del KIDS-52 por tipo de establecimiento.

\begin{tabular}{lccc} 
& $\begin{array}{c}\text { Municipal } \\
(\mathbf{2 6 6 )}\end{array}$ & $\begin{array}{c}\text { Subvencionado } \\
\mathbf{( 2 7 1 )}\end{array}$ & $\begin{array}{c}\text { Particular } \\
\mathbf{( 1 6 5 )}\end{array}$ \\
& $\mathbf{X} \mathbf{\text { DE }}$ & $\mathbf{D E}$ & $\mathbf{D E}$ \\
\hline Bienestar físico & $53.28+28.18$ & $55.42+31.66$ & $51.99+23.76$ \\
Bienestar Psicológico & $58.98+24.78$ & $57.09+22.48$ & $56.61+15.73$ \\
Estado emocional y ánimo & $35.18+27.71$ & $35.30+24.47$ & $32.35+22.55$ \\
Autopercepción & $44.81+18.72$ & $44.05+14.48$ & $42.62+10.52$ \\
Autonomía & $51.59+19.72$ & $50.45+12.75$ & $51.87+17.41$ \\
Relación con los padres y vida familiar & $59.05+43.88$ & $58.28+31.95$ & $54.30+19.88$ \\
Recursos económicos & $46.18+11.43$ & $45.99+11.27$ & $48.88+11.48$ \\
Amigos y apoyo social & $59.07+33.14$ & $59.36+32.90$ & $58.83+29.65$ \\
Entorno escolar & $58.22+30.14$ & $59.61+36.39$ & $53.61+21.58$ \\
Bullying & $24.23+9.25$ & $23.45+11.14$ & $23.13+11.75$
\end{tabular}

\section{TABLA 6}

\section{Correlaciones entre las dimensiones de KIDS-52 y EAT-26.}

\begin{tabular}{|c|c|c|c|c|c|c|c|c|}
\hline \multirow[b]{3}{*}{ KIDS - 52} & \multicolumn{8}{|c|}{ EAT -26 } \\
\hline & \multicolumn{2}{|c|}{ RT } & \multicolumn{2}{|c|}{ CO } & \multicolumn{2}{|c|}{ DIE } & \multicolumn{2}{|c|}{ BUL } \\
\hline & $\mathbf{r}$ & $\mathbf{r} \square$ & $\mathbf{r}$ & $\mathbf{r} \square$ & $\mathbf{r}$ & $\mathbf{r} \square$ & $\mathbf{r}$ & $\mathbf{r} \square$ \\
\hline Bienestar Físico & .03 & & .03 & & .05 & & .05 & \\
\hline Bienestar psicológico & -.07 & & -.01 & & -.06 & & .02 & \\
\hline Estado emocional y ánimo & .11 & & -.02 & & $.08(*)$ & & .05 & \\
\hline Autopercepción & .06 & & .06 & & .09 & & .06 & \\
\hline Autonomía & -.09 & & -.02 & & -.05 & & -.05 & \\
\hline Relación con los padres y vida familiar & -.01 & & .00 & & .03 & & -.01 & \\
\hline Recursos económicos & $-.12(*)$ & .01 & .01 & & -.04 & & -.05 & \\
\hline Amigos y apoyo social & .00 & & -.02 & & .03 & & .06 & \\
\hline Entorno escolar & $.14(*)$ & .02 & $.11(* *)$ & .01 & $.09(*)$ & .00 & .07 & \\
\hline Bullying & $.19(* *)$ & .04 & .03 & & $.12(* *)$ & .01 & $.14(*)$ & .02 \\
\hline
\end{tabular}


subvencionados sólo en la variable recursos económicos $(\mathrm{F}(2.70)=3.82, \mathrm{p}<.05)$.

\section{Calidad de vida y actitudes alimentarias}

La tabla 6 muestra las relaciones entre las dimensiones evaluadas de CV y las actitudes alimentarias. Solo algunas dimensiones evaluadas de la CV correlacionaron con las actitudes alimentarias. Se encontró una correlación positiva entre el estado emocional y ánimo y la conducta de dieta $(\mathrm{r}(701)=.08)$, una relación inversa entre recursos económicos y el riesgo total $(\mathrm{r}(701)=$ -.12), una correlación positiva entre entorno escolar y el riesgo total $(\mathrm{r}(701)=.14)$, control oral $(\mathrm{r}(701)=.11)$ y dieta $(\mathrm{r}(701)=.09)$ y una correlación también positiva entre la dimensión bullying y el riesgo total $(\mathrm{r}(701)=.19)$, dieta $(r(701)=.12)$ y bulimia $(r(701)=.14)$. Cabe destacar que en todas estas la medida del tamaño del efecto reporta una baja magnitud de la relación.

Considerando las diferencias encontradas tanto en $\mathrm{CV}$ como en conductas alimentarias entre hombres y mujeres, se analizó la relación existente estratificándose por sexo (tabla 7). La dimensión Bullying fue la única variable que correlacionó con una CAR en ambos sexos, en el caso de las mujeres con el riesgo total $(\mathrm{r}(376)=.17)$, dieta $(\mathrm{r}(376)=.15)$ y bulimia $(\mathrm{r}(376)=.19)$, y en el caso de los hombres sólo con dieta $(\mathrm{r}(326)=.12$,). En las mujeres se encontró además una correlación entre entorno escolar y riesgo total $(\mathrm{r}(376)=.13)$ y control oral $(\mathrm{r}(376)=.12$,$) .$

\section{TABLA 7}

Correlaciones entre las dimensiones de Kids-52 y EAT-26 segmentadas por sexo.

\begin{tabular}{|c|c|c|c|c|c|c|c|c|}
\hline & \multicolumn{2}{|c|}{ RT } & \multicolumn{2}{|c|}{ CO } & \multicolumn{2}{|c|}{ DIE } & \multicolumn{2}{|c|}{ BUL } \\
\hline & $\mathbf{r}$ & $\mathbf{r} \square$ & $\mathbf{R}$ & $\mathbf{r}$ & $\mathbf{r}$ & $\mathbf{r} \square$ & $\mathbf{r}$ & $\mathbf{r} \square$ \\
\hline \multicolumn{9}{|l|}{ Mujeres } \\
\hline Bienestar físico & .01 & & .06 & & -.01 & & -.03 & \\
\hline Bienestar psicológico & -.06 & & .00 & & -.09 & & .01 & \\
\hline Estado emocional y ánimo & .05 & & -.04 & & .08 & & .05 & \\
\hline Autopercepción & .07 & & .08 & & .05 & & .02 & \\
\hline Autonomía & -.09 & & -.02 & & -.09 & & -.09 & \\
\hline Relación con los padres y vida familiar & .03 & & 03 & & .04 & & .04 & \\
\hline Recursos económicos & -.07 & & -.01 & & -.08 & & -.04 & \\
\hline Amigos y apoyo social & .01 & & .06 & & -.03 & & .03 & \\
\hline Entorno escolar & $.13(*)$ & .02 & $.12(*)$ & .01 & .09 & & .10 & \\
\hline Bullying & $.17(* *)$ & .03 & .08 & & $.15(* *)$ & .02 & $.19(* *)$ & .03 \\
\hline \multicolumn{9}{|l|}{ Hombres } \\
\hline Bienestar físico & $.14(*)$ & .02 & .04 & & $.14(*)$ & .02 & $.12(*)$ & .01 \\
\hline Bienestar psicológico & -.00 & & -.03 & & .00 & & .04 & \\
\hline Estado emocional y ánimo & .05 & & -.02 & & .07 & & .04 & \\
\hline Autopercepción & .08 & & .03 & & .07 & & .10 & \\
\hline Autonomía & .01 & & -.01 & & .02 & & .00 & \\
\hline Relación con los padres y vida familiar & -.01 & & -.04 & -.00 & .03 & & & \\
\hline Recursos económicos & -.01 & & .03 & & -.01 & & -.06 & \\
\hline Amigos y apoyo social & .05 & & -.08 & & .09 & & .09 & \\
\hline Entorno escolar & .08 & & .07 & & .07 & & .02 & \\
\hline Bullying & .09 & & -.02 & & $.12(*)$ & .01 & .09 & \\
\hline
\end{tabular}


En los hombres se encontró una relación entre la dimensión de bienestar físico y el riesgo total $(\mathrm{r}(326)=.14)$, la dieta $(\mathrm{r}(326)=.14)$ y la bulimia $(\mathrm{r}(326)=.12)$. Cabe destacar que en todas estas relaciones la medida del tamaño del efecto reporta una baja magnitud.

Las dimensiones bullying y entorno escolar fueron las únicas variables que se relacionaron con una actitud alimentaria en ambos rangos de edad (tabla 8). En el caso del bullying, en el grupo de 10 a 11 años correlacionó positivamente con la bulimia $(\mathrm{r}(328)=.16)$, en tanto en el grupo de 12 a 13 años lo hizo con la dimensión dieta $(\mathrm{r}(372)=.13)$, bulimia $(\mathrm{r}(372)=.10)$, y riesgo total $(r(372)=.13)$. El dominio entorno escolar correlacionó positivamente con la dimensión control oral $(\mathrm{r}(328)=.12)$, en el grupo de 10 a 11 años, y con dieta $(\mathrm{r}(372)=.12)$ y riesgo total $(\mathrm{r}(372)=.12)$, en el caso del rango de 12 y 13 años.

En el rango de edad entre 12 y 13 años, se encontraron correlaciones significativas entre las dimensiones bienestar psicológico y dieta $(\mathrm{r}(372)=-.11)$, estado emocional y dieta $(\mathrm{r}(372)=.14)$ y riesgo total $(\mathrm{r}(372)=.10)$, autopercepción con bulimia $(\mathrm{r}(372)=.11)$ y riesgo total $(\mathrm{r}(372)=.11)$, autonomía y bulimia $(\mathrm{r}(372)=-.10)$ y finalmente recursos económicos con dieta $(\mathrm{r}(372)=-.12)$ y riesgo total $(\mathrm{r}(372)=-.12)$.

Cabe destacar que en todas, la medida del tamaño del efecto reporta una baja magnitud de la relación.

Al estratificar el análisis por el tipo de estableci-

\section{TABLA 8}

Correlaciones entre las dimensiones de la escala KIDS-52 y EAT-26 según rango de edad.

\begin{tabular}{|c|c|c|c|c|c|c|c|c|}
\hline & \multicolumn{2}{|c|}{$\mathrm{CO}$} & \multicolumn{2}{|c|}{ DIE } & \multicolumn{2}{|c|}{ BUL } & \multicolumn{2}{|c|}{ RT } \\
\hline & $\mathbf{r}$ & r $\square$ & $\mathbf{r}$ & $\mathbf{r} \square$ & $\mathbf{r}$ & $\mathbf{r} \square$ & $\mathbf{r}$ & $\mathbf{r} \square$ \\
\hline \multicolumn{9}{|l|}{10 a 11 años } \\
\hline Bienestar físico & .03 & & .08 & & .05 & & .075 & \\
\hline Bienestar psicológico & -.04 & & -.04 & & .01 & & -.037 & \\
\hline Estado emocional y ánimo & -.05 & & .05 & & .05 & & .027 & \\
\hline Autopercepción & .07 & & .04 & & .02 & & .058 & \\
\hline Autonomía & -.04 & & -.02 & & .00 & & -.025 & \\
\hline Relación con los padres y vida familiar & -.04 & & -.02 & & .00 & & -.025 & \\
\hline Recursos económicos & -.04 & & -.02 & & .00 & & -.025 & \\
\hline Amigos y apoyo social & -.01 & & .04 & & .07 & & .037 & \\
\hline Entorno escolar & $.12(*)$ & .01 & .04 & & .06 & & .087 & \\
\hline Bullying & .01 & & .10 & & $.16(* *)$ & .03 & .105 & \\
\hline \multicolumn{9}{|l|}{12 a 13 años } \\
\hline Bienestar físico & .01 & & -.03 & & .02 & & -.01 & \\
\hline Bienestar psicológico & .01 & & $-.11(*)$ & .01 & .02 & & -.06 & \\
\hline Estado emocional y ánimo & .00 & & $.14(* *)$ & .02 & .05 & & $.10(*)$ & \\
\hline Autopercepción & .06 & & .09 & & $.11(*)$ & .01 & $.11(*)$ & .01 \\
\hline Autonomía & .00 & & -.08 & & $-.10(*)$ & .00 & -.07 & \\
\hline Relación con los padres y vida familiar & .05 & & .02 & & -.01 & & .03 & \\
\hline Recursos económicos & -.06 & & $-.12(*)$ & .01 & -.05 & & $-.12(*)$ & .01 \\
\hline Amigos y apoyo social & -.03 & & .01 & & .04 & & -.00 & \\
\hline Entorno escolar & .07 & & $.12(*)$ & .01 & .04 & & $.12(*)$ & .01 \\
\hline Bullying & .04 & & $.13(* *)$ & .02 & $.10(*)$ & .00 & $.13(*)$ & .02 \\
\hline
\end{tabular}




\section{TABLA 9}

Correlaciones entre las dimensiones de la escala KIDS-52 y EAT-26 según tipo de establecimiento.

\begin{tabular}{|c|c|c|c|c|c|c|c|c|}
\hline & \multicolumn{2}{|c|}{$\mathrm{CO}$} & \multicolumn{2}{|c|}{ DIE } & \multicolumn{2}{|c|}{ BUL } & \multicolumn{2}{|c|}{ RT } \\
\hline & $\mathbf{r}$ & ra & $\mathbf{r}$ & $\mathbf{r} \square$ & $\mathbf{R}$ & $\mathbf{r} \square$ & $\mathbf{r}$ & $\mathbf{r} \square$ \\
\hline \multicolumn{9}{|l|}{ Municipal } \\
\hline Bienestar físico & -.01 & & .00 & & .11 & & .03 & \\
\hline Bienestar psicológico & .01 & & -.12 & & -.01 & & -.07 & \\
\hline Estado emocional y ánimo & -.01 & & $.15(*)$ & .02 & .06 & & .11 & \\
\hline Autopercepción & .09 & & .03 & & .00 & & .06 & \\
\hline Autonomía & -.03 & & -.07 & & -.10 & & -.09 & \\
\hline Relación con los padres y vida familiar & .01 & & .01 & & -.07 & & -.01 & \\
\hline Recursos económicos & -.12 & & -.10 & & -.04 & & $-.12(*)$ & .01 \\
\hline Amigos y apoyo social & -.03 & & -.01 & & .09 & & .00 & \\
\hline Entorno escolar & .11 & & .11 & & .07 & & $.14(*)$ & .02 \\
\hline Bullying & .10 & & $.19(* *)$ & .03 & .11 & & $.19(* *)$ & .03 \\
\hline \multicolumn{9}{|l|}{ Subvencionado } \\
\hline Bienestar físico & .02 & & .07 & & -.00 & & .05 & \\
\hline Bienestar psicológico & -.02 & & .02 & & .09 & & .03 & \\
\hline Estado emocional y ánimo & -.03 & & .03 & & .01 & & .01 & \\
\hline Autopercepción & .04 & & .06 & & $.12(*)$ & .01 & .08 & \\
\hline Autonomía & .00 & & -.02 & & -.05 & & -.02 & \\
\hline Relación con los padres y vida familiar & -.06 & & .07 & & .06 & & .03 & \\
\hline Recursos económicos & .05 & & -.02 & & -.05 & & -.01 & \\
\hline Amigos y apoyo social & .04 & & .03 & & .03 & & .04 & \\
\hline Entorno escolar & .04 & & .06 & & .07 & & .07 & \\
\hline Bullying & .01 & & $.13(*)$ & .02 & $.17(* *)$ & .03 & $.13(*)$ & .02 \\
\hline \multicolumn{9}{|l|}{ Particular } \\
\hline Bienestar físico & .11 & & .09 & & .04 & & .11 & \\
\hline Bienestar psicológico & -.04 & & -.10 & & -.06 & & -.09 & \\
\hline Estado emocional y ánimo & -.04 & & .05 & & .08 & & .04 & \\
\hline Autopercepción & .05 & & .13 & & .06 & & .12 & \\
\hline Autonomía & .01 & & -.03 & & .07 & & .01 & \\
\hline Relación con los padres y vida familiar & .15 & & -.07 & & -.05 & & .01 & \\
\hline Recursos económicos & $.17(*)$ & .03 & .02 & & -.02 & & .07 & \\
\hline Amigos y apoyo social & -.13 & & .10 & & .03 & & .02 & \\
\hline Entorno escolar & $.28(* *)$ & .08 & .11 & & .01 & & $.18(*)$ & .03 \\
\hline Bullying & -.02 & & .03 & & .11 & & .03 & \\
\hline
\end{tabular}

** La correlación es significativa al nivel 0,01 (bilateral); * La correlación es significativa al nivel 0,05; CO=Control Oral; DIE= Dieta; BUL= Bulimia; RT= Riesgo total; $r=$ Coeficiente de correlación de Pearson; $\mathrm{r}^{2}=$ Medida del tamaño del efecto. 
miento, ninguna de los dominios de calidad de vida correlacionó con una actitud alimentaria en los tres tipos de instituciones a la vez (tabla 9).

Solo para los dominios entorno escolar y bullying se encontró relación con la actitud alimentaria en dos tipos de establecimientos a la vez. En el caso del entorno escolar, este correlacionó significativamente con la dimensión riesgo total $(\mathrm{r}(265)=-.14)$ en los establecimientos municipales y asimismo con riesgo total $(\mathrm{r}(164)=-.18)$ y control oral $(\mathrm{r}(164)=.28)$, en el caso de los establecimientos particulares. La dimensión bullying correlacionó con dieta $(\mathrm{r}(265)=.19)$ y riesgo total $(\mathrm{r}(265)=.19)$ en los establecimientos municipales y dieta $(\mathrm{r}(270)=.13)$, bulimia $(\mathrm{r}(270)=.17)$ y riesgo total $(\mathrm{r}(270)=.13)$ en los establecimientos subvencionados.

En los establecimientos municipales además se encontraron correlaciones significativas entre las dimensiones estado emocional y ánimo con dieta $(r(265)=.15)$ y en recursos económicos con el riesgo total $(\mathrm{r}(265)=-.12)$.

En los establecimientos subvencionados se encontró relación entre autopercepción y bulimia $(\mathrm{r}(270)=.12)$ $\mathrm{y}$ en los establecimientos particulares entre recursos económicos y control oral (r(164)=.17). Cabe destacar que en todas estas relaciones la medida del tamaño del efecto reporta una baja magnitud.

\section{DISCUSIÓN}

Los resultados siguen aportando evidencia de una mayor prevalencia de CAR en las mujeres, incluyendo el grupo etario de la preadolescencia. Posibles explicaciones se plantean desde la construcción de los roles de género que inciden en la percepción de la imagen corporal, en donde a las mujeres se les invita y se les recuerda constantemente "el poder que posee la apariencia física y el valor de la delgadez", y que el comer poco es más femenino (3). Los datos con relación al riesgo en grupos de edad menores (10 y 11 años), aportan evidencia a la disminución cada vez mayor del riesgo de TCA a grupos de menores edades, sin disminuir la prevalencia de riesgo en los otros rangos de edad (5). Cabe destacar que actualmente los preadolescentes comienzan a preocuparse a más temprana edad por su imagen corporal, debido a que está asociada a una mayor popularidad y éxito, rechazando la gordura.

Los resultados sugieren que la preocupación por el control del peso y la imagen corporal lleva a los preadolescentes de entre 12 a 13 años a desarrollar mayor CAR, afectando su CV, en particular en las dimensiones: bienestar psicológico, bullying, entorno escolar, estado emocional y ánimo, autopercepción, autonomía y recursos económicos. En la edad escolar aparecería la preocupación por la imagen corporal, la que se asocia con popularidad, inteligencia y éxito y se rechaza la gordura. Esta situación se observa con mayor frecuencia en las mujeres, las que centran su ideal en un cuerpo delgado, en la prevalente focalización en la apariencia externa y la trascendencia de esta figura en el éxito social, tendencia que se acentúa durante la adolescencia (12). De manera similar, estudios reportan que a medida que avanza la edad disminuye la media de la $\mathrm{CV}$ en todas las dimensiones. Es posible que los infantes perciban de mejor manera su apariencia física que los y las adolescentes, dado que aun no comienza el período de desarrollo y con esto los problemas generados con el cuerpo por el surgimiento de las características sexuales secundarias (13).

Así, los adolescentes con TCA han descrito una disminución de la $\mathrm{CV}$ en todas las dimensiones como el bienestar físico, psicológico y social (14).

En la investigación realizada se encontró una relación significativa, aunque de baja magnitud, entre el bienestar físico y el riesgo a padecer un TCA, dieta y bulimia en participantes de sexo masculino. Con respecto a esto, los preadolescentes que presentan mayores niveles de actividad física, energía y buena condición física están más preocupados por estar delgados, evitando alimentos que engordan y pensando más en los alimentos que consumen. Se ha planteado que la mayoría de los adolescentes están más preocupados por su imagen corporal y apariencia que por cualquier otro aspecto de si mismos, presentando en algunos casos esfuerzos obsesivos por controlar el peso, a través de dietas, ejercicio físico en exceso o conductas purgativas, siendo incluso poco probable un problema real de peso (15).

En relación a la dimensión entorno escolar y bullying de la CV se observó una relación significativa y de baja magnitud con las dimensiones de la Escala de Actitudes alimentarias, por lo que se puede inferir que la CV esta fuertemente marcada por el contexto escolar. En términos evolutivos, en este periodo cobra mayor importancia la relación con los pares en el colegio, buscando mayor aceptación, por lo que se le da más atención a la imagen corporal, muchas veces incurriendo en actitudes alimentarias de riesgo. Por otro lado, el bullying se hace presente en este entorno, generando sentimientos de rechazo en quienes presentan estándares físicos alejados de la figura corporal ideal, teniendo más riesgo a desarrollar un TCA.

Estudios han encontrado la existencia de un factor facilitador, que genera su transmisión entre los escolares, actuando aquellos con trastornos alimentarios como vectores, produciendo entre sus pares una imitación en la preocupación por el alimento y la silueta corporal (2).

En la dimensión estado emocional y ánimo de la CV 
se encontró una relación significativa y de baja magnitud con dieta y riesgo a desarrollar un TCA, coincidiendo con la literatura revisada la que afirma la presencia de experiencias negativas, los estados de ánimo depresivos y sensaciones de estrés en personas con desordenes alimentarios. En cuanto esto, estudios señalan que "en los desórdenes alimentarios, la depresión suele manifestarse asociada a la anorexia, el insomnio, la tristeza, el aislamiento, el llanto, la anhedonia, los problemas de autoconcepto, incluyendo la autocrítica que se extiende desde la autoestima hasta la imagen corporal, acompañada de culpa, vergüenza y desesperanza" (16). Se ha señalado que una trastorno de la conducta alimentaria esta acompañado por un deterioro de la CV, especialmente en el ámbito del bienestar emocional (17).

En relación a la autonomía de la $\mathrm{CV}$, esta se relaciona de manera inversa y de baja magnitud con la dimensión bulimia de la escala de actitud alimentaria, por lo que se infiere que los preadolescentes que presentan mayor preocupación por la comida e indicadores de bulimia, tendrían una menor autonomía relacionada con la toma de decisiones en su tiempo de ocio. Estudios refieren que generalmente, los trastornos alimenticios se dan en jóvenes que están más propicias a quedarse estancadas en la niñez por su temor a crecer y a manejar la independencia que eso implica. Este cuerpo que inevitablemente transmite que ya se ha dejado la infancia, representa para muchas de estas jóvenes, una realidad intolerable, o bien afecta a chicas que perciben el temor de sus padres a que se vuelvan autónomas, y sufren en realidad por la falta de estímulos de parte de ellos para animarse a entrar en el mundo adulto. Este miedo a crecer se manifiesta mediante síntomas orgánicos hasta los más complejos trastornos alimenticios, sin proponerlo transforman así su cuerpo en el lugar donde sí podrán sentirse "en control" de la situación y lo convierten en el símbolo y la fuente de autonomía y eficacia personal. El resultado es el retarde de asumir responsabilidades temidas, pero también el perderse disfrutar prerrogativas propias de una nueva etapa (18).

Se encontró una relación significativa, inversa y de baja magnitud entre la dimensión recursos económicos de la $\mathrm{CV}$ con la dimensión riesgo total a padecer un TCA, en familias con menos recursos económicos existe mayor tendencia a desarrollar conductas alimentarias de riesgo, por lo que en familias con mayores recursos económicos este riesgo disminuye debido a que cuentan con mayores posibilidad de acceder a una alimentación saludable. Lo encontrado coincide con estudios de la OMS en países industrializados, en donde los adolescentes de familias menos acomodadas tienden a reportar una salud más pobre y síntomas más frecuentes, en cambio los adolescentes de familias más adinerados suelen tener dietas más sanas y mayor actividad física (15). Sin embargo, algunos estudios refieren que una de características predominantes de los desórdenes alimentarios es que se presentan generalmente en pacientes de un nivel socioeconómico medio-alto (2), aun cuando se ha generalizado a todos los niveles socioeconómicos.

Es importante consignar que este estudio ha permitido evaluar el nivel de impacto de cada una de las variables estudiadas en relación al riesgo y conductas alimentarias en cada uno de los diversos dominios que componen la Calidad de vida en preadolescentes de diversos niveles socioeconómicos, lo que permitirá mayor certeza a la hora de planificar intervenciones destinadas a mejorar la $\mathrm{CV}$ de preadolescentes con estos trastornos.

Se considera necesario, continuar con estudios en relación a la $\mathrm{CV}$ y actitudes alimentarias, en especial en preadolescentes, ya que existe escasa información al revisar la literatura existente, tanto en Chile como en otros países. Además, estos estudios podrían enfocarse en la construcción de programas de intervención dirigidos a preadolescentes, ya sea en contextos educaciones, familiares o de salud, que contribuyan a prevenir actitudes alimentarias de riesgo a padecer un TCA, con el objetivo de mejorar el bienestar y la CV de esta población que ha sido escasamente considerada. Por otro lado, se podría contribuir al desarrollo de políticas públicas en salud que promuevan la $\mathrm{CV}$ en niños y adolescentes, considerando que son el grupo más alejado de los centros de atención primaria en salud y/o como grupo objetivo de investigaciones o programas de intervención.

En cuanto a las limitaciones que presenta este estudio, podemos destacar la escasez de datos estadísticos en cuanto a los preadolescentes, puesto que no son un grupo de mayor interés en investigaciones o programas de intervención. Otra de las limitaciones de la investigación es que al ser un estudio de tipo correlacional no se sabe cual es la direccionalidad de las variables, por lo que los resultados no indican si existe una relación causa - efecto entre éstas. Además, al no poder controlar las variables no es posible saber si existe alguna otra variable no observada que podría estar influyendo o causando la relación.

\section{RESUMEN}

Con un diseño transversal, se evaluó la relación existente entre la calidad de vida y las conductas alimentarias en preadolescentes de entre 10 a 13 años de edad. Se analizaron datos de 702 participantes (media de edad de 11.5 años, 375 mujeres y 327 hombres) recogidos a través de los instrumentos KIDSCREEN-52, para medir la calidad de vida y el EAT-26, para evaluar las conductas 
alimentarias. Se encontró que la preocupación por el control del peso y la imagen corporal están relacionadas con una menor calidad de vida de los preadolescentes, en particular en las dimensiones de bienestar físico, bullying, entorno escolar, estado emocional y ánimo, autopercepción, autonomía y recursos económicos. Los hallazgos encontrados, así como la evidencia que estas conductas se presentan cada vez a más temprana edad, incidiendo en el bienestar y la calidad de vida de los y las preadolescentes, ameritan el desarrollo de intervenciones preventivas tempranas en el campo de las conductas alimentarias.

Palabras clave: Calidad de vida, conductas alimentarias, preadolescencia.

Dirigir la correspondencia a:

Profesor

Alfonso Urzúa M.

Escuela de Psicología

Universidad Católica del Norte

Avenida Angamos 0610

Antofagasta_Chile

Email:alurzua@ucn.cl

\section{BIBLIOGRAFÍA}

1. Ludueña A, Olson J., Pasco A. Promoción de la salud y calidad de vida entre madres de preadolescentes. Una etnografía enfocada. Rev Latinoamericana Enfermagem 2005; 13: 1127-34.

2. Behar R, Alviña M, González T, Rivera N. Detección de actitudes y/o conductas predisponentes a trastornos alimentarios en estudiantes de enseñanza media de tres colegios particulares de viña del mar. Rev Chil Nutr. 2007; 34: 240-49.

3. López P, Molina Z, Rojas L. Influencia del género y la percepción de la imagen corporal en las conductas alimentarias de riesgo en adolescentes de Mérida. Anales Venezolanos Nutr. 2008; 21: 85-90.

4. Rodríguez B, Oudhof H, González A. López I, Unikel C, Becerril M. Conductas alimentarias de riesgo: prevalencia en mujeres de nivel superior del estado de México. Rev Científica Electrónica de Psicol. 2008; 6: 159-70.

5. Correa V, Zubarew G, Silva P, Romero M. Prevalencia de riesgo de trastornos alimentarios en adolescentes mujeres escolares de la Región Metropolitana. Rev Chil Pediatr. 2006; 77: 153-160.

6. Behar R. Trastornos del hábito de comer en adolescentes: aspectos clínicos y epidemiológicos. Rev Méd Chile 1998; 126: 1085 -92.
7. Behar R, Alviña M, Medinelli A, Tapia P. Trastornos de la conducta alimentaria en estudiantes de la carrera de nutrición y dietetica. Rev Chil Nutr 2007; 34(4): 298-306.

8. Urzúa A. Calidad de Vida relacionada con la salud: aspectos conceptuales. Rev Méd Chil. 2010; 138: $358-65$.

9. Ravens-Sieberer U, Gosch A, Rajmil L, Erhart M, Bruil J, Duer W, Auquier P, Power M, Abel T, Czemy L, Mazur J, Czimbalmos A, Tountas Y, Hagquist $\mathrm{C}$, Kilroe $\mathrm{J} \&$ the European KIDSCREEN Group. KIDSCREEN-52 quality-of-life measure for children and adolescents. Expert Review Pharmacoeconomics Outcomes Res. 2005; 5: 353-64.

10. Tebe A, Berra S, Herdman M, Aymerich M, Alonso J, Rajmil L. Fiabilidad y validez de la versión española del Kidscreen-52 para población infantil y adolescente. Med Clínica 2008; 130: 650 - 54.

11. Gandarillas, A. Zorrilla, B. Sepúlveda, A. Trastornos del comportamiento alimentario: Prevalencia de casos clínicos en mujeres adolescentes de la Comunidad de Madrid. Servicio Promoción Salud 2003; 85, 11-120.

12. Marín V. Trastornos de la conducta alimentaria en escolares y adolescentes. Rev Chil Nutr. 2002; 29: 86-91.

13. Urzúa A., Cortés E., Prieto L., Vega S., Tapia K. Autoreporte de la calidad de vida en niños y adolescentes escolarizados. Rev Chil Pediatr 2009; 8: $238-44$.

14. Herpertz B, Wille N, Holling H, Vloet T, RavensSieberer U. \& the BELLA study group . Disordered eating behaviour and attitudes, associated psychopathology and health-related quality of life: results of the BELLA study. Eur Child Adolesc Psychiatry 2008; 17:82-91.

15. Duskin R, Papalia D, Wendkos S. Psicología del desarrollo: de la infancia a la adolescencia. México: McGraw-Hill. 2005.

16. Behar R, Barahona M, Iglesias B, Casanova D. Trastorno depresivo, ansiedad social y su prevalencia en trastornos de la conducta alimentaria Rev Chil Neuro-Psiquiatr. 2007; 45: 211-20.

17. Doll H, Petersen S, Stewart S. Eating disorders and emotional and physical well-being: Associations between student self-reports of eating disorders and quality of life as measured by the SF-36. Quality Life Res. 2005; 14: 705-17.

18. Crispo R, Guelar D. Adolescencia y trastornos del comer. Barcelona: Gedisa. 2001. 